\title{
Analysis on the Basketball Match Video based on the Image Segmentation Algorithm
}

\author{
Jiewei Yang ${ }^{\mathrm{a}}$ \\ Department of Physical Education,Huanghuai University, HenanZhumadian China 463000 \\ alfy4387@126.com
}

Keywords: Image segmentation algorithms, Basketball match, Video.

\begin{abstract}
With the development of the camera, video camera, infrared and various sensors such as visual information capture device with the technology, it has become a simple question that the visual information is obtained, but now in the era of information explosion, how to capture the interest of visual information in part become an important research direction, which is one of the most fundamental problems in the digital image processing. The image processing and segmentation is the bottom and the most basic work, only the good image segmentation result can perform image analysis work. This paper proposes the extracting method of the basketball match video games based on image segmentation algorithm.
\end{abstract}

\section{Introduction}

With the development of Internet technology and digital media technology, the analysis of match video gradually becomes a new research hotspot. Found in the mass match video data of their favorite video also has become a big user demand, the traditional management and retrieval method using data from a text identifier is hard to meet the needs of users, this makes the rapid rise of content-based video retrieval method and technology is gradually used in all kinds of match video analysis and retrieval. At the same time, the sports and the study of the motion object segmentation and tracking of the video is the focus of the present study, mainly based on the characteristics of the different kinds of sports, establish the corresponding video analysis and the research methods. In terms of basketball game, is characterized by movement violent video game objects are relatively fixed, sharp and focused on objects with the basketball athletes.

\section{The Image Segmentation Techniques}

Image segmentation technology is accompanied by the generation of digital image processing, as military, remote sensing, computer vision technology in industry, agriculture and other areas of application is becoming more and more widely, in the computer vision technology, the image acquisition plays a role of link to the image to understand the process of image segmentation technology, the importance of starting to show. However, image segmentation is a basic and complex cross subject, and physics, physiology, and there are many subjects such as mathematics, although after decades of research, but still there is no universal method, this is because image segmentation is a "sick" problem and evaluation standard are hard to quantify, each method has its characteristic, also has limitations, and algorithm complexity segmentation result is difficult to do both. Based on the characteristics of digital image segmentation method can be roughly divided into three broad categories: based on threshold segmentation, based on the edge of the segmentation, segmentation based on region. Objects in the image gray level, often with the cluster characteristics, namely the object's internal grey value often changed little, while the grey value between objects is not the same, based on threshold image segmentation method by setting gray-level threshold image segmentation into several parts. Classic threshold segmentation algorithm has maximum entropy segmentation method, the minimum error method, and so on, its development has experienced from the single threshold to the change of threshold value. Method has simple theory, based on threshold segmentation the characteristics of fast speed, but most of the method without using spatial information of images, so in the face of complex image segmentation effect is not good. 
The image segmentation method based on region as a piece of the collection of pixels, pixels with similar characteristics by clustering algorithm together to form a continuous area, this method accord with characteristics of human visual perception. Classic region segmentation algorithm is with k-means clustering method, region growing method, watershed algorithm, etc. Based on region segmentation method can obtain a closed area, in the absence of prior knowledge also can obtain very good segmentation results, but often high algorithm complexity, time consuming.

In recent years, puts forward a new segmentation method is mainly to incorporate new theory with the traditional segmentation method in order to in the segmentation result and efficiency two aspects to obtain. In terms of threshold segmentation, the most important thing is to determine the threshold of, namely how to rapidly and accurately obtain the best threshold. Focus on rapid threshold value in remote sensing image threshold segmentation algorithm selection and noise processing problems, the introduction of a neighborhood space and gray similarity measure factor for noise processing, and then to the most between-cluster variance as the hybrid leapfrog algorithm fitness function, through hybrid leapfrog algorithm local search and global information exchange to quickly determine the global optimal threshold image segmentation. Can see threshold algorithm research focused on the quick look most thresholds which on the one hand.

\section{Analysis on the Match Video Semantic}

The characteristics of match video. We will from the structure of match video viewers, rules, and high-level semantic three aspects are discussed on the characteristics of match video: from the point of viewers, we will be roughly divided into ordinary audience, professional audience, 3 coaches or professional class, for different audiences, our research focus is not the same.

1) The general audience: watching sports may only be a form of recreation or relax, how to quickly provide instant game, good game, the shooting scene, such as soccer Ace in the tennis ball, and other key is the study of this part of the audience; 2) The professional audience: here we define "professional audience" for the be fond of sports. They make up the ratings of the main body, and the game will have a choice, but competition is various, the energy, the time is limited, how to do the most efficient way to provide them with the required video game is becoming a hot spot of research; 3) The coach or professionals: making competition strategy through a video game, or research the technical characteristics of athletes, they are more paying attention to the details in the video. How to automatically track the location of the athletes, according to the semantic retains the effective data is the main content of the study group.

From the structure rules: 1) the late prophase shoots and edit more regularly. Due to the limitation of the venue, the limited number of camera used in sports, and the Angle is relatively fixed. Common are: panoramic lens (or long), the venue located in the central screen, shooting game in the works; Close-up, general is outstanding shooting athlete or a part of the body. Different position and Angle, different types of lens provides viewers with important semantic information, also provides important reference basis for video analysis. 2) The rules of the game are relatively fixed. Different sport has its inherent rules, such as the NBA game points, four sections, each section 12 minutes, 15 minutes rest between two, three, 2 minutes off the rest of the internodes, etc. Use of the known rules for match video analysis has brought great convenience. From senior video semantic: sports in the existing rules are clear semantic definition, such as: basketball game dunk, shooting, free throws, the definition of a football game shooting, the definition of free-kicks, corners, etc., clear semantic definition can help reduce subjectivity and the convenience of semantic analysis, lay a foundation for crossing the "semantic gap".

The match video data redundancy. Different viewers, the focus of attention may be all the same, but for most of the audience, perhaps the most attention is the events of the game, based on this the paper put forward the match video (SRD), the concept of redundancy data, here we define SRD: sports game is not recorded in the effective time of game footage. Such as: all competitions, pause time of break. 


\section{The Establishment of Basketball Objects Extraction Method based on the Image Segmentation Algorithm}

In order to achieve the object of basketball extraction, first to basketball objects for selected video frames, to determine the selected target for foreground object. In order to get the basketball objects, precise segmentation, the background modeling method is used to change the selected object of basketball as a separation of foreground from the background, then USES the Crab Cut algorithm to basketball object more precise segmentation.

The basketball selection of object and background modeling. Based on single frame video image object of basketball as a foreground to background modeling with the background, the background and the concept of outlook, outlook refers to the hypothesis that the background for the static condition, the movement of any meaningful objects for outlook, namely in the basketball match in the video can be as a prospect, the corresponding game scene as the background, the basic idea of modeling is that from the current frame image to extract the foreground, the purpose is to make the background more close to the background of the current video frame, it is using the current frame and background of the current frame in the video sequence weighted average to update the background.

Study on basketball match video analysis has some research results at home and abroad, such as, through to the basketball stadium in video color distribution statistics, the video game into the game with suspend video clips; Basketball in the video object segmentation and tracking, and to establish trajectories. This paper proposes a basketball object extraction method based on image segmentation, through the study of the background modeling of basketball match video frame, choose basketball as foreground segmentation to extract object. Extraction of basketball object can be used for video clip retrieval system tracking target, at the same time the basketball object segmentation and extraction is the core part of basketball match video clip retrieval framework.

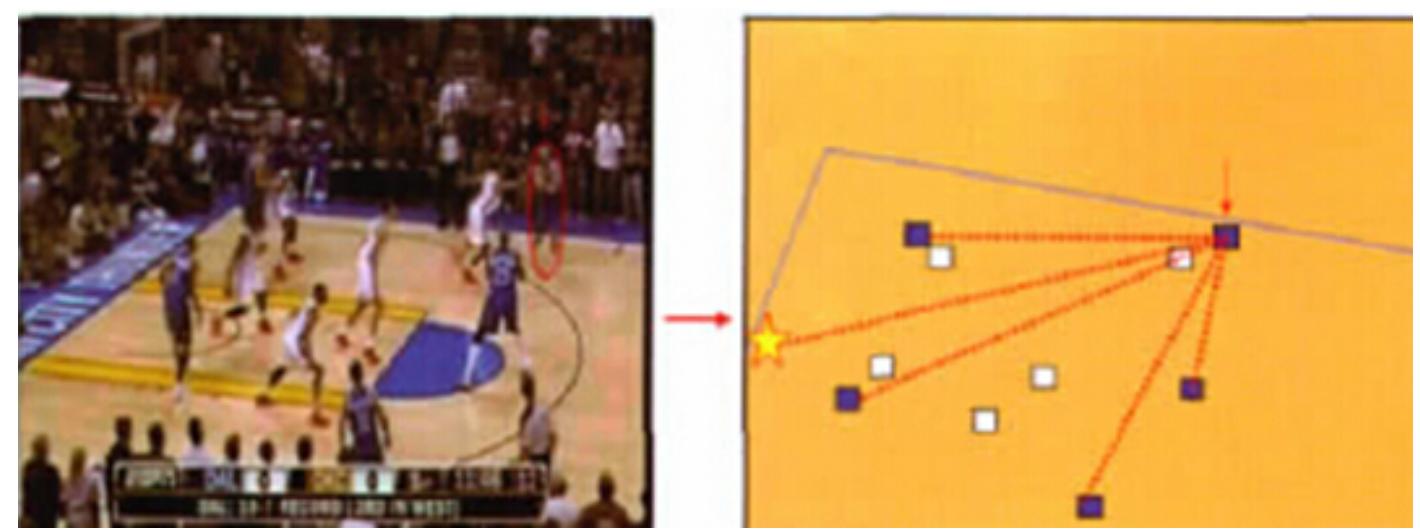

Fig. 1 The Basketball match video

To calculate the main color average range. We take 20 different types of the basketball video games, and repeat Step1-Step5, that can obtain a landlord colors mean $\overline{c o l} \_s e q$, according to the

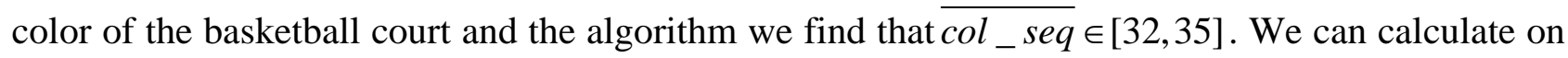
the basis of the basketball match video main colors mean accurate results and get the main sequence.

Table1 Average of main regional color

\begin{tabular}{cccc}
\hline $\begin{array}{c}\text { The basketball match } \\
\text { video }\end{array}$ & col_seq & $\begin{array}{c}\text { The basketball match } \\
\text { video }\end{array}$ & $\overline{c o l \_s e q}$ \\
\hline Video 1,2 & 32,34 & Video 11,12 & 35,34 \\
Video 3,4 & 33,34 & Video 13,14 & 33,34 \\
Video 5,6 & 35,33 & Video 15,16 & 33,33 \\
Video 7,8 & 32,33 & Video 17,18 & 32,33 \\
Video 9,10 & 34,34 & Video 19,20 & 34,34 \\
\hline
\end{tabular}


Algorithm steps. Step1. Artificial given basketball video segmentation, capture panoramic camera video similar to figure 2 period of $20 \mathrm{~s}$, and all of them as experiment frame sequence image frames;

Step2. To regional segmentation of experimental frames used way, keep the middle area.

Step3. Calculate all the frames used in HSV color components in the space the sum of the histogram $H_{\text {sum }}$.

$$
H_{\text {sum }}=\sum_{i=1}^{n} H\left(I_{i}\right)
$$

$\mathrm{n}$ is the total number of image frames in the 20s video.

Step4. Calculate the sum of all pixels Total $_{\text {sum }}$ in the $H_{\text {sum }}$.

$$
\text { Total }_{\text {sum }}=\sum_{i=1}^{k} H_{\text {sum }}
$$

$\mathrm{K}$ is for image frames color quantization level. Here we will attune (original h component of HSV space for grade 360) quantitative level of 40 , namely $k=40$. The experiment proved that after the quantitative stadium has good color clustering.

Step5. Calculate the average color.

$$
\overline{c o l \_s e q}=\frac{\sum_{i=1}^{n}\left(\operatorname{col}_{\text {max }_{i}}\left(\text { Total }_{\text {sum }}\right)\right)}{n}
$$

$\mathrm{Col}_{\text {max }_{i}}\left(\right.$ Total $\left._{\text {sum }}\right)$ is the $\mathrm{i}$ in the value of the corresponding color in the Total ${ }_{\text {sum }}$, the main color sequence number is 5 , so $n=5$, the largest of the five values corresponding to the average value in the Total $_{\text {sum }}$.

\section{Summary}

With the growing popularity of the TV commercial, the sports broadcasting time is longer and longer, and the actual game time is limited, so rapid and accurate detection of sports in the redundant data, keep effective video game, no matter the ordinary audience or television's staff are highly beneficial.

\section{References}

[1] L. Xie, P. Xu, S. Chang, A Divakaran, H Sun. Structure analysis of soccer video with domain knowledge and hidden Markov models. Pattern Recognition Letters. 2012(10)

[2] W. Lu, J. Little. Tracking and Recognizing Actions at a Distance. European Conference on Computer Vision Workshop on Computer Vision Based Analysis in Sport Environments. 2012(08)

[3] H. Miyamori. Improving Accuracy in Behavior Identification for Content-based Retrieval by Using Audio and Video Information. IEEE International Conference on Pattern Recognition. 2012(12)

[4] X.F. Tong, L.Y. Duan and C.S. Xu. Local motion analysis and its application in video based swimming style recognition. Proceedings of the 18th International Conference on Pattern Recognition. 2012(12). 